\title{
Advantages of 3D Echocardiography in the Diagnosis of Mitral Valve Endocarditis and its Cardiac Complications
}

\section{Cacciapuoti $\mathbf{F}^{*}$}

\author{
Ambulatory of Echocardiography of Internal Medicine Department, "L. Vanvitelli" University of Campania, Italy
}

*Corresponding author: Cacciapuoti F, Ambulatory of Echocardiography of Internal Medicine Department, “L. Vanvitelli” University of Campania, Piazza L. Miraglia, 280100, Naples, Italy, E-mail: fulvio.cacciapuoti@gmail.com Citation: Cacciapuoti F (2018) Advantages of 3D Echocardiography in the Diagnosis of Mitral Valve Endocarditis and its Cardiac Complications. J Cardio Disord Therapy 1: 104

Article history: Received: 28 February 2018, Accepted: 28 March 2018, Published: 02 April 2018

\section{Abstract}

Background: Endocarditis (E) (i.e. an infection of the endocardial surface) can involve heart valves (native or prosthetic) or the mural endocardium and can induce numerous and heavy complications.

Methods: The Duke diagnostic criteria are used to make the diagnosis of infective (E). These combine the clinical, pathologic, microbiologic and echocardiographic aspects. Echocardiography plays a key role and was considered as a major Duke criteria for diagnosis of infective E. Particularly, ultrasonic method has assumed an increasing importance for the diagnosis of valves' $\mathrm{E}$.

Results: We only evaluated the importance of 3D-Echocardiography (3D-E), both from trans-thoracic (TTE) and trans-esophageal (TEE) view, in the detection of native mitral valve vegetation's by E, their pre-surgical complications and post-surgical aspects.

Conclusions: 3D-TTE and TEE, significantly increased the diagnosis of mitral infective E and vegetations, even when the blood culture are negative. In particular, 3D-E (TTE and TEE) appear useful in defining pre-surgical findings of mitral E, the exact location of leflets' vegetations, their size and shape and the post-procedural assessment. Thus, 3D-E findings represent an effective advancement in comparison with previous echocardiographic techniques.

Keywords: Mitral valve infective endocarditis; 3D echocardiography; cardiac complications and surgical correction.

List of abbreviations: IE: Infective Endocarditis; NVE: Native Valve Endocarditis; PVE: Prosthetic Valve Endocarditis; M-Mode: Mono-dimensional Mode; 3D-E: 3 Dimensional Echocardiography; TTE: Trans-Thoracic Echocardiography; TEE: Trans.Esophageal Echocardiography

\section{Introduction}

Infective Endocarditis (IE), also called bacterial endocarditis, is a life-threatening disease due to the infection of the endocardial surface of the heart, which may involve one or more heart valves or the mural endocardium or possible septal defect [1,2]. Mitral and aortic valves are most frequently involved, while tricuspid limbs are less implicated. Finally, IE of pulmonary valve is an extremely uncommon entity, accounting for less $2 \%$ of all reported cases. The common causative micro-organisms are streptococci, staphylococci or fungi. Several cardiac and extracardiac complications may occur during IE, as embolic events, heart failure, perivalvular extension, splenomegaly, pericardial and/or pleural effusion, arrhythmias and others and cardiovascular symptoms related to different IE locations [3]. Various types of IE can be recognized:

- Native valve endocarditis (NVE), acute and subacute;

- Prosthetic valve endocarditis (PVE), early and late;

- Intravenous drug abuse endocarditis.

- Other types of endocarditis include pacemaker IE and nosocomial IE

Acute NVE concerns normal valves and has an aggressive course. Subacute NVE involves abnormal valves, it is less aggressive and may extend over many months [4]. In turn, PVE may involve both mechanical and bioprosthetic valves. Mitral valve is more susceptible to both NVE and PVE than the aortic valve [5]. Contrarily that of heart valves, the incidence of IE on pacemaker devices is constantly increased in the last decade. This can induce a high rate of severe complications. Recently Carrasco et al. found almost $10 \%$ of IE on pacemaker devices in the last 6 years. This condition often requires removal of devices in spite of therapy [6]. 
IE may be suspected in different clinical situations, as heart failure, cerebral embolism, unexplained fever with or without regurgitant heart murmur, night sweats, signs of systemic illness, new conduction disturbance and others. The suspect of IE must be confirmed by hemocultures and/or echocardiographic findings. On the subject, the Duke diagnostic criteria are used to define IE diagnosis (Table 1). These combine clinical, microbiologic, pathologic and echocardiographic characteristics differentiated in major and minor criteria [7]. At the present time, echocardiography is considered as major Duke criteria and has assumed a crucial role in the IE diagnosis, particularly when blood cultures are negative [8,9]. In particular, ultrasonic technique does not only consent the diagnosis of IE, but it is also useful for follow-up, possible cardiac complications (documented by an oscillating or non-oscillating intracardiac masses on a valve or on supporting structures; peri-valvular or myocardial abscesses; dehiscences of prosthetic valves; new-onset valvular regurgitation) and medical and/or surgical management. But, recent evidences have reported encouraging results for CT and PET in the diagnosis of IE and some complications, as abscesses. The addition of CT/PET at a major Duke criterion significantly increased its sensitivity and specificity.

\begin{tabular}{|c|c|}
\hline \multicolumn{2}{|c|}{ DUKE CRITERIA } \\
\hline MAJOR & MINOR \\
\hline${ }^{*}$ Positive hemocultures & ${ }^{*}$ Intravenous drugs abuse \\
\hline${ }^{*}$ Positive echocardiographic findings & ${ }^{*}$ Fever $>38{ }^{\circ} \mathrm{C}$ \\
\hline${ }^{*}$ New valvular regurgitation & ${ }^{*}$ Heart disease \\
\hline & ${ }^{*}$ Minor echo findings (eg. deformed valve but no vegetations) \\
\hline
\end{tabular}

Table 1: Duke criteria for noninvasive diagnosis of infective endocarditis

In this report, we only illustrated the aspects and the exact location of mitral valve vegetations, their main cardiac complications and the results obtained by medical or surgical interventions, seen at three-dimensional Echocardiography (3D-E), performed both from trans-thoracic (TTE) and trans-esophageal (TEE) approach [10-12]. The different roles of Echocardiography in the evaluation of IE are summarized in Table 2.

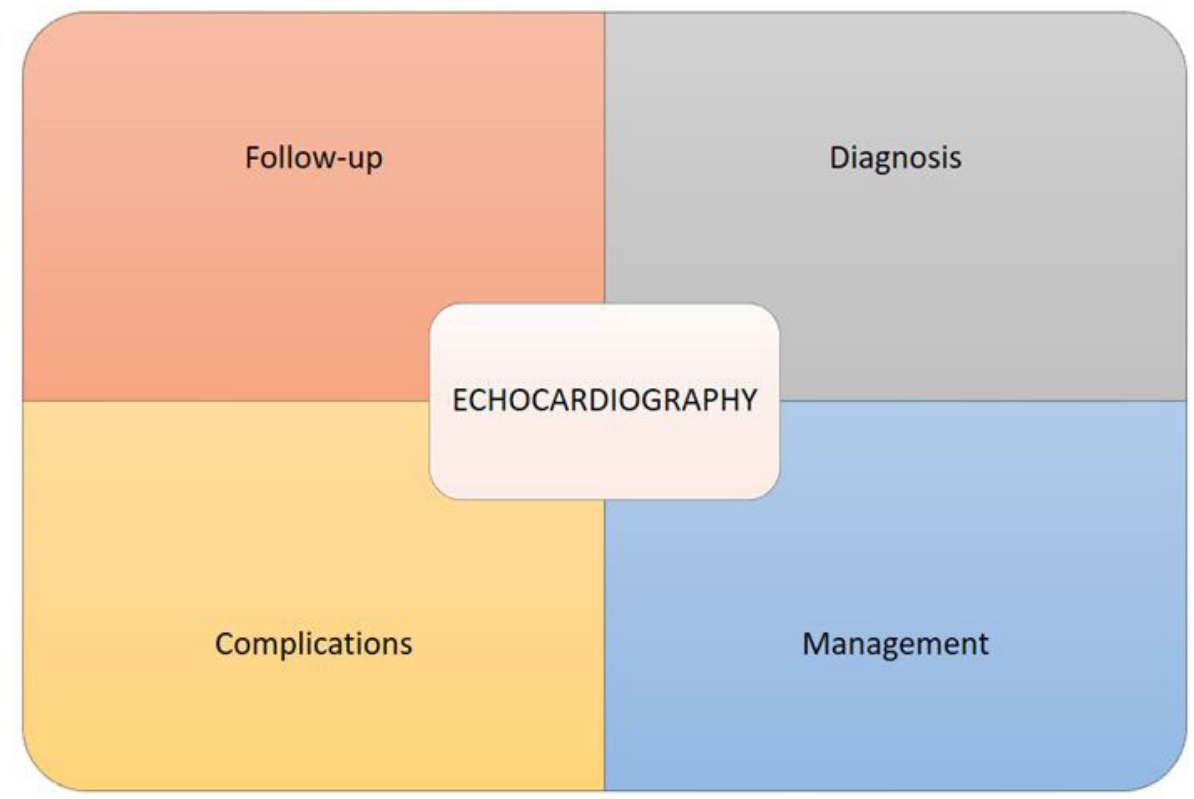

Table 2: Different roles of Echocardiography in the diagnosis, follow-up, complications and management of Infective Endocarditis

\section{Echocardiography}

Echocardiography is the only noninvasive method today used, allowing direct visualization of endocarditis-induced lesions. The combined M-mode and 2-dimensional trans-thoracic echocardiography significantly increased the detection rate of mitral valve IE and its complications, in comparison to M-mode alone introduced 30 years ago. In addition, whereas 2D-TEE reveals signs of IE on both native and prosthetic valves, the advent of 3D-TEE enables superior pre-operative anatomic evaluation for complications of IE, with consequent improvement in surgical planning.

\section{Three-dimensional echocardiography}

\section{D-TTE}

As previous affirmed, even though the sensitivity and specificity of 2D-trans-thoracic echocardiography (2D-TTE) are high, the improved spatial orientation of 3D-TTE enables more precise and complete examination of intracardiac anatomy. In fact, 3D-TTE provides a choice acquisition mode, including multiplane, real time live 3D, full-volume 3D, zoom 3D and 3D Color Doppler. 
This ultrasound mode enabled the visualization of cardiac structures and valvular pathology, particularly mitral valve's vegetations [13]. It must also added that 3D-TTE has many advantages over 2D-TTE including improved visualization of complex shapes and spatial relations among cardiac structures. In addition, 3D-TTE has an incremental role in evaluating valvular anatomic feature, volumetric quantification, pre-surgical planning, intra-procedural guidance and post-procedural assessment of valvular heart disease. In addition, 3D-TTE shows the precise location of vegetations, their size and relationship with the mitral limbs, both in short and long-axis views (Figures $1 \mathrm{~A}$ and $1 \mathrm{~B}$ ). It also provides accurate information for medical and surgical decision making $[14,15]$. In agreement, comparative studies have confirmed that size of vegetations is more accurately measured on real-time $3 \mathrm{D}$ TTE in comparison with 2D-TTE [16].
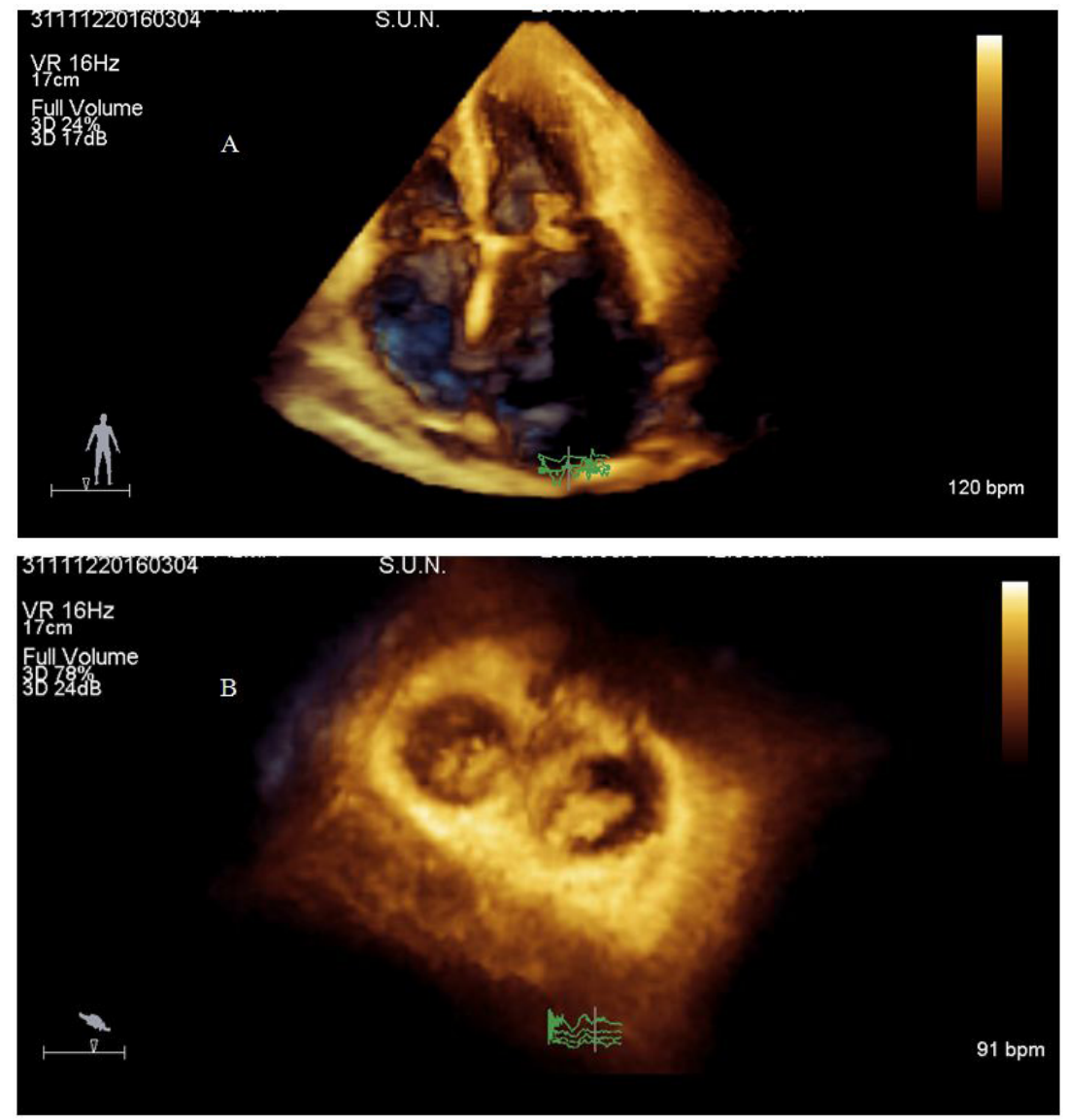

Figure 1: A-3D-TTE. Apical four-chambers view showing a mitral vegetation located between both mitral leaflets and protruding in left atrial and ventricular cavities. B-Same vegetation seen in short-axis approach

\section{D-TEE}

The following development of high quality real-time three dimensional trans-esophageal echocardiography (3D-TEE) further improved the diagnosis of mitral valve endocarditis and its complications [17]. The technique seems to be particularly important in patients whom 3D TTE fails to provide imaging quality. In particular, it is able in those having advanced obesity, chest deformity, or enphisema or in patients subjected to artificial respiration. Specifically, 3D-TEE consents to visualize the mitral anterior leaflet from $78 \%$ to $100 \%$ and the posterior leaflet of $100 \%$ [18]. Referring to the mitral IE, 3D-TEE has proven to be the powerful, convincing method for understanting the anatomy of mitral valve. The sensitivity for the diagnosis of vegetation's is about $85-$ 90\% for 3D TEE versus 75\% for 3D TTE. In addition, color Doppler 3D TEE has been valuable to identify the location of the regurgitant orifice and the severity of the mitral regurgitation. It was also pointed out that 3D-TEE facilitates the visualization of the full extension and motion of the IE vegetations in one view from any derived angle. Specifically, mitral vegetations appear as long and narrow structures that are usually attached to mitral anterior leaflet. On the contrary, 2D-TEE fails to provide sufficient informations regarding the relationship among vegetations, prosthesis, and adjacent structures [19]. Nevertheless, 2D-TEE should be performed in the majority of patients with suspected IE, because of its better image quality and better sensitivity, particularly for the diagnosis of peri-valvular involvement [12]. However, 3D (both in TTE and TEE modes) is often performed when the clinical suspicion of IE is high and M mode and two-dimensional echocardiography is inconclusive or negative. It must also underline that 3D TEE increases the visualization of mitral valve perforation (Figure 2) in comparison with other echocardiographic techniques [18]. Furthermore, 3D TEE is superior to 3D TTE in identifying the structural complications of IE (as leaflet rupture or flail), in locating and evaluating prosthetic dehiscence, in to visualize perivalvular abscesses, valvular perforation, fusion of commissures and pericardial effusion. This method has been shown to provide additional information in the evaluation of PVE [19]. From this 
point of view, 3D TEE images are not limited to 2D planar views, enabling valvular visualization of angles not previously possible. This possibility must be reported to the acquisition of wide-angled, full-volume data sets and the ability to manipulate and crop images, deep anatomical structures can be clearly displayed (Figure 3) [20]. With reference to post-operative evaluation of mitral valve repair or replacement, 3D TEE has been shown to facilitate visualization of the entire structure of artificial valve. Finally, the method has become indispensable for non-surgical procedures, such as "edge to edge" mitral repair and transcatheter closure of paravalvular leaks. Referring to this issue, 3D-TEE appeared useful to show an immediate reduction in the antero-posterior diameter of the mitral annulus in mitral regurgitation, after MitraClip procedure [21].

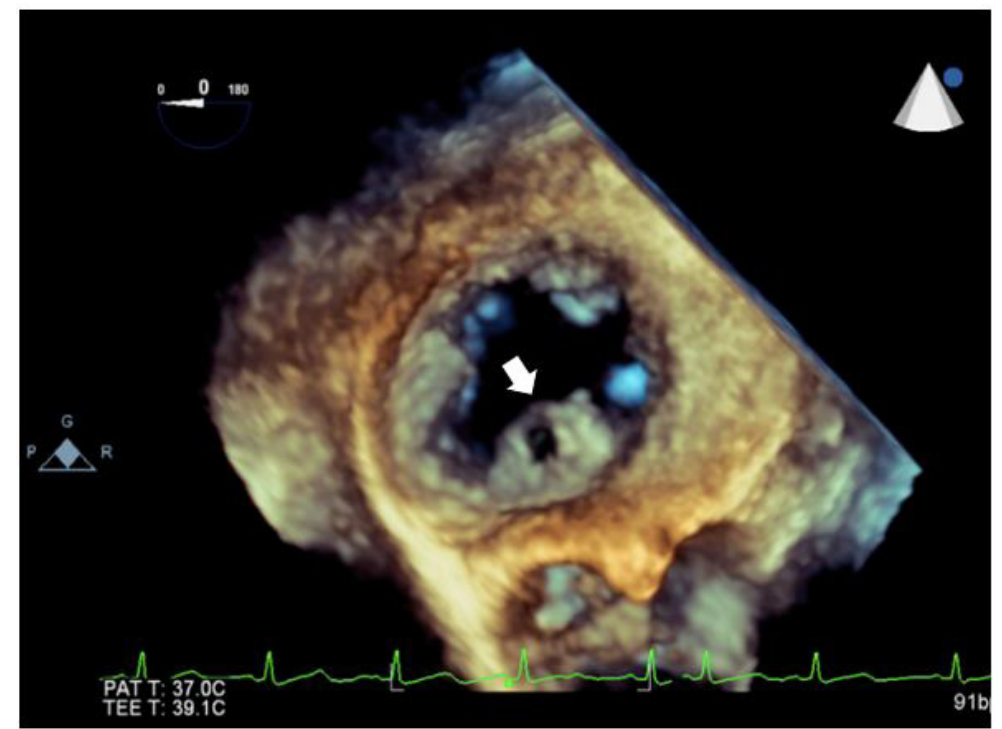

Figure 2: 3D-TEE-Mitral limb perforation in a patient having mitral infective endocarditis

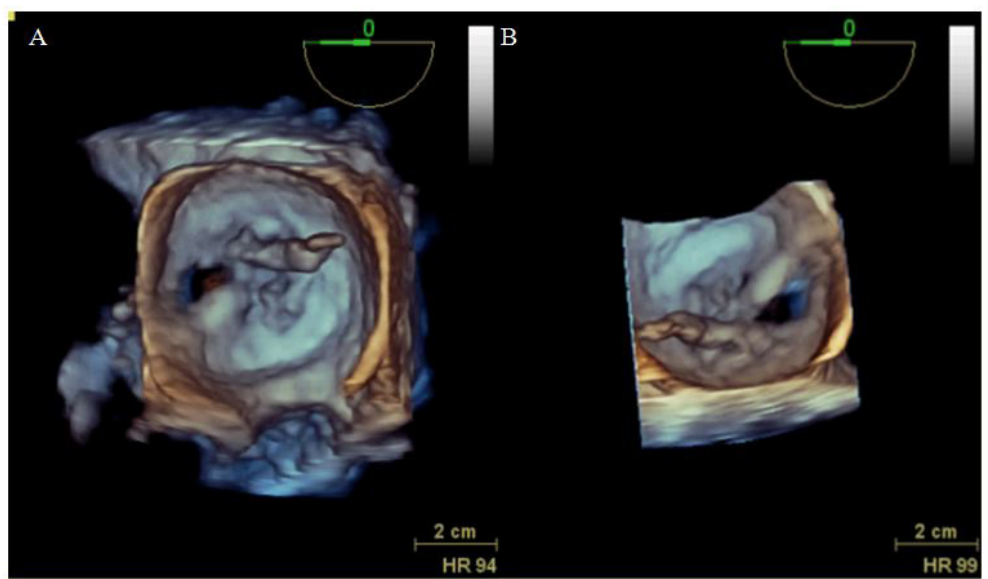

Figure 3: 3D-TEE-Mitral valve vegetation seen from left atrial side (A - B). The shape, the dimensions and the location are clearly evidenced

\section{Conclusive remarks}

Echocardiography has assumed an increasing importance in the diagnosis of IE, especially located at mitral leaflets. The development of 3D-TTE and its combination with 3D TEE have progressively improved the non-invasive detection of cardiac valves' vegetation's and the complications of IE, such as aneurysm, perforation, prolapse or papillary muscle rupture. Specifically, 3D TEE zoom modality can provide "en-face" views of both mitral and aortic valves. By convention, the mitral valve can be rotated and viewed from the left atrial perspective. Dehiscence site can be also identified, with special attention to their location, shape, size and area. Using multiplanar imaging it is possible to quantify the area of dehiscence. In addition, full-volume acquisition provides wide angle images with higher temporal resolution. After data acquisition, data set can be rotated, manipulated and cropped to obtain exposure of para-valvular leaks, confirmed with the use of 3D color flow [21]. 3D TTE and 3D TEE were also employed for the follow-up and prognostic assessment of IE. It's follow-up (time-evolution) must be performed in early, intermediate and late phase (hospitalization, the course of illness, surgical intervention). Prognosis must be defined by the findings associated with the vegetations, such as regurgitation or obstruction of the mitral valve (native or prosthetic), systolic para-valvular jets, significant reduction of ejection fraction, premature mitral closure and others [22]. Conclusively, in the presence of suspect IE, 3D TTE, and 3D TEE assumed a significant role in the non-invasive diagnosis of that, and must be performed when clinical criteria are indicative of IE but, blood cultures are negative and M-mode and 2D-TTE examinations are not diagnostic. Therefore, at the present time 3D-Echocardiography appears to be as complementary to 2D-Echocardiography, even though further improvements in image quality, higher temporal and spatial resolution and software are foreseen in the near future. 


\section{References}

1. Karchmer AW (2005) Infective endocarditis. Braunwald's heart disease. A textbook of Cardiovascular Medicine ( $7^{\text {th }}$ edn) WB Saunders Co $1633-58$.

2. Karchmer AW (2005) Infective endocarditis. Harrison's Principles of Internal Medicine. (16 $6^{\text {th }}$ edn) Mc Graw-Hill; 731-40.

3. Horskotte D, Follath F, Gutschik E, Lengyel M, Oto A, et al. (2004) Guidelines on prevention, diagnosis and treatment of infective endocarditis executive summary, the task force on infective endocarditis of the European Society of Cardiology. Eur. Heart J 25: 267-76.

4. Nishimura RA, Carabello BA, Faxon DP, Freed MD, Lyle BW, et al. (2008) ACC/AHA 2008-Guideline update on valvular heart disease focused update on infective endocarditis. A report of the American College of Cardiology/American Heart Association Task Force on practice guidelines. Circulation 118: 887-96.

5. Baddour LM, Wilson LM (2005) Infections of prosthetic valves and other cardiovascular devices: intravascular devices. Mandell GL, Bennett JE, Dolin R, ed. Mandel, Douglas and Bennett's Principles and Pratice and Infectious Diseases (5th edn) Philadelphia, Pa: Elservier 1022-44.

6. Carrasco F, Anguita M, Ruitz M, Castillo JC, Delgado M, et al. (2016) Clinical features and changes in epidemiology of infective endocarditis on pacemaker devices over a 27 year (1987-2013). Europace 18: 836-41.

7. Durack DT, Lukes AS, Bright DK (1994) New criteria for diagnosis of infective endocarditis; utilization of specific echocardiographic findings. Duke Endocarditis Service. Am J Med 96: 200-9.

8. Evangelista A, Gonzales-Alujas MT (2004) Echocardiography in infective endocarditis. Heart 90: 614-7.

9. Mugge A, Daniel WG, Lichtlen PR (1989) Echocardiography in infective endocarditis; reassessment of prognostic implications of vegetation size determined by the transthoracic and the transesophageal approach. J Am Coll Cardiol 14: 631-8.

10. Reynolds HR, Jogen MA, Tunick PA, Kronzon I (2003) Sensitivity of transthoracic versus transesophageal echocardiography for the detection of native valve vegetations in the modern era. JASE 16: 67-70.

11. Shapiro ScM, Young E, De Guzman S, Ward J, Chiu C (1994) Transesophageal echocardiography in diagnosis of infective endocarditis. Chest 105: 377-82.

12. Habib G, Hoen B, Tornos P, Thuny F, Prendergast B, et al. (2009) ESC Committee for Pratice Guidelines (CPG). Guidelines on the prevention, diagnosis, and treatment of infective endocarditis (new version 2009): The Task Force on the prevention, diagnosis and treatment of Infective Endocarditis of the European Society of Cardiology (ESC). Eur. Heart J 30: 2369-43.

13. Hansalia S, Biswas M, Dutta R, Hage FC, Hsiung M, et al. (2009) The value of live/real time three-dimensional transesophageal echocardiography in the assessment of valvular vegetations. Echocardiography 26: 1264-73.

14. Tsang W, Weinert L, Kronzon I, Lang RM (2011) Ecocardiografia tridimensional en la evaluation de las valvulas protesicas. Rev. Esp Cardiol 64: 1-7.

15. Perez de Isla L, Zamorano J, Malangatana G, Almeria C, Rodrigo JL, et al. (2005) Usefulness of real-time-three dimensional echocardiography in the assessment of infective endocarditis-Initial experience. J Ultrasound Med 24: 231-3.

16. Asch FM, Breganki SP, Panza JA, Weissman NJ (2006) Real-time three dimensional echocardiography evaluation of intracardiac masses. Echocardiography 23: 218-24.

17. Liu YW, Tsai WC, Lin CC, Hsu CH, Li WT, et al. (2009) Usefulness of real-time three-dimensional echocardiography for diagnosis of infective endocarditis. Scand. Cardiovasc J 43: 318-23.

18. Schwalm SA, Sugeng L, Roman J, Jeevanandum V, Lang RM (2004) Assessment of mitral leaflet perforation as a result of infective endocarditis by 3 - dimensional real-time echocardiography. JASE 17: 919-22.

19. Berdejo J, Shibayama K, Harada K, Tanaka J, Mihara H, et al. (2014) Evaluation of vegetation size and its relationship with embolism in infective endocarditis. A real-time 3-dimensional transesophageal echocardiography study. Circ Cardiovasc Imaging 7: 149-54.

20. Flachskamp FA, Wouters PF, Edvardsen T, Evangelista A, Habib G, et al. (2014) Recommentations for trans-esophageal echocardiography EAVI update 2014. Eur J Cardiol Imaging 15: 353-65.

21. Hidalgo F, Mesa D, Ruiz M, Delgado M, Rodriguez S, et al. (2016) Effects of mitral annulus remodeling following MitraClip procedure on the reduction of functional mitral regurgitation. Rev Esp Cardiol 69: 1020-5.

22. Vrettou AR, Zacharoulis A, Lerakis S, Kremastinos DT (2013) Revealing infective endocarditis complications by echocardiography: the value of real-time 3D trans-esophageal echocardiography. Hellenic J Cardiol 54: 147-9.

23. Habib G, Lancellotti P, Antunes MJ, Bongiorni MG, Casalta JP, et al. (2015) 2015 ESC Guidelines for the management of infective endocarditis. The Task Force for the management of infective endocarditis of the European Society of Echocardiography. Eur Heart J 36: 3075-128. 